\title{
Soziale Netzwerke und gesundheitliche Ungleichheiten im jungen und mittleren Erwachsenenalter
}

\section{Holger von der Lippe und Olaf Reis}

\section{Überblick}

- Im Lebenslauf junger und mittlerer Erwachsener dürfte es enge Zusammenhänge zwischen sozialen und gesundheitlichen Ungleichheiten, biografischen Übergängen, sozialen Netzwerken und Gesundheitsverhalten geben. Viele dieser Zusammenhänge sind noch nicht hinreichend erforscht.

- Lebenslauftheorien der Entwicklung in sozialen Beziehungen Erwachsener werden berichtet und in ihrer Passung für Gesundheit und Gesundheitsverhalten diskutiert.

- Exemplarische Studien zur gesundheitlichen Bedeutung sozialer Netzwerke in biografischen Anpassungssituation junger und mittlerer Erwachsener (wie z. B. Paarbildung, Scheidung, Auszug der erwachsenen Kinder) werden vorgestellt.

- Nach aktueller Forschungslage ist ein Mediatoreffekt sozialer Netzwerke für den Einfluss sozialer Ungleichheiten auf Gesundheit wahrscheinlich.

- Die Forschungslage ist nicht gefestigt, weitere empirische wie theoretische Anstrengungen werden als notwendig erachtet.

H. von der Lippe $(\bowtie)$

Berlin, Deutschland

E-Mail: holger.vonderlippe@medicalschool-berlin.de

O. Reis

Rostock, Deutschland

E-Mail: olaf.reis@med.uni-rostock.de 
„Krankheit, Verlust des Gleichgewichts, meint nicht nur einen medizinischbiologischen Tatbestand, sondern auch einen lebensgeschichtlichen und gesellschaftlichen Vorgang. “

(Gadamer 1965)

\section{$1 \quad$ Einleitung: der thematische und paradigmatische Rahmen sozialer Netzwerke und gesundheitlicher Ungleichheiten im jungen und mittleren Erwachsenenalter}

$\mathrm{Zu}$ Beginn seiner einschlägigen Einführung in „Social Networks and Health“ formuliert Valente (2010) bereits ein zentrales Postulat, das auch für dieses Kapitel grundlegend sein wird. Er konstatiert, dass die wissenschaftliche Beschäftigung mit sozialen Beziehungsnetzen im Gesundheitskontext stets in einem interdisziplinären Zugang zum Gegenstand sowie unter Beachtung einer Entwicklungsbzw. Lebensspannenperspektive erfolgen müsse. Dies ist eine anspruchsvolle Forderung, bedeutet sie doch zum einen, dass man Netzwerke und Gesundheit zugleich als ein soziologisches Thema (z. B. ungleich verteilt, schichtabhängig), als ein psychologisches Thema (z. B. verhaltens- und persönlichkeitsabhängig) sowie unter einigen anderen disziplinären Perspektiven mehr (z. B. epidemiologisch, biologisch-physiologisch, gesundheitswissenschaftlich) verstehen kann und sollte (Interdisziplinarität des Gegenstandes). Und zum anderen bedeutet Valentes Postulat auch, dass die Frage nach Netzwerkeffekten im Gesundheitskontext kaum allgemein für „,den Menschen“ gestellt werden kann, sondern für unterschiedliche Altersphasen oder Lagen im Lebenslauf zu unterschiedlichen Ergebnissen kommen wird (Lebensspannenperspektive).

Auch wir werden in unserem Kapitel dieses sehr grundlegende Postulat Valentes aufgreifen und uns mit der Gesundheits- und Ungleichheitsrelevanz von Netzwerken aus einer psychologischen wie soziologischen Lebensspannenperspektive beschäftigen. Dabei richten wir ein besonderes Augenmerk auf wechselseitige Interaktionen zwischen Gesundheit, sozialer Ungleichheit und Netzwerken im Kontext biografischer Übergänge, welche den Lebenslauf Erwachsener entscheidend mitprägen (Lang et al. 2006), und fokussieren ausschließlich das junge und mittlere Erwachsenenalter - hier grob definiert als die Alterspanne von etwa 20 bis 60 Jahren. Einen zweiten Schwerpunkt unserer Darstellung wird die Entwicklungspsychologie der Lebensspanne ausmachen (Brandtstädter und Lindenberger 2007), deren konzeptuelles Verständnis von Netzwerken und Gesundheit bislang noch recht wenig Eingang in die Forschung gefunden hat. 
In diesem ersten Kapitel werden wir zunächst die bereits angedeuteten unterschiedlichen disziplinären Perspektiven und Paradigmen einführen, die sich mit dem Thema Netzwerke und gesundheitliche Ungleichheiten in dieser Lebensphase befassen. Hier werden zentrale Begriffe wie Lebenslauf und Transition, Beziehungen und Netzwerk, Gesundheits- und Risikoverhalten sowie soziale und gesundheitliche Ungleichheiten definiert. In Abschn. 2 werden dann Theorien dargestellt, die Wechselwirkungen zwischen den genannten Konzepten beschreiben. Hier legen wir einen Schwerpunkt insbesondere auf sozial- und entwicklungspsychologische Theorien, welche die genannten Konstrukte mit dem Erwachsenenalter als Lebensphase verknüpfen.

In den dann folgenden Abschnitten werden wir den Forschungsstand zu den Zusammenhängen zwischen sozialer und gesundheitlicher Ungleichheit (Abschn. 3), Netzwerken und Gesundheit (Abschn. 4) sowie Ungleichheit, Netzwerken und Gesundheit (Abschn. 5) zusammentragen. Um das junge und mittlere Erwachsenenalter als eigene Lebensphase anschaulich zu thematisieren, gehen wir auf drei Transitionen genauer ein: den Beginn einer festen Partnerschaft, Scheidungs- und Trennungsereignisse sowie den Auszug der Kinder aus dem Elternhaus. Diese biografischen Übergänge sind einerseits typische Übergänge der betrachteten Altersphase, und andererseits gibt es in der Literatur die Sichtweise, dass derartige normative oder nicht-normative Transitionen als vulnerable Zeiten individueller Entwicklung verstanden werden können, in denen mögliche Netzwerkeffekte auf Gesundheit besonders deutlich werden (Lang et al. 2006). Wir schließen mit einem Fazit und den Desiderata für zukünftige Forschung (Abschn. 6).

\subsection{Disziplinäre Perspektiven auf Netzwerke und gesundheitliche Ungleichheiten im jungen und mittleren Erwachsenenalter}

Die Altersspanne des jungen und mittleren Erwachsenenalters gilt aus einer soziologischen Ungleichheitsperspektive heraus seit jeher als eine sehr bedeutsame und dynamische Lebensphase fortwährender individueller Veränderung. Im Altersabschnitt zwischen 20 und 60 Jahren durchläuft der Einzelne verschiedene biografische Übergänge (sogenannte Transitionen wie z. B. Auszug aus dem Elternhaus, Umzüge, Heirat, berufliche Übergänge, Scheidung etc.), die allesamt das Potenzial haben, soziale, psychische und gesundheitliche Unterschiede zu vergrößern oder zu verkleinern. Eine Transition bezeichnet dabei den Wechsel einer Person von einer Lebenslage hin zu einer anderen mit einer markanten 
Vorher-nachher-Distinktion, die meist mit einem definierten Übergang im sozialen Status und/oder der sozialen Identität einhergeht. Solche Transitionen gehen besonders häufig mit Veränderungen in der Struktur und Funktion sozialer Beziehungen und Netzwerke einher.

Auch in der gesundheitswissenschaftlichen Forschung sind Verläufe von subjektiven wie objektiven Gesundheitsunterschieden im jungen und mittleren Erwachsenenalter zunehmend in das Blickfeld geraten. Aus der subjektiven Gesundheitsperspektive fällt die Altersspanne beispielsweise durch den U-förmigen Verlauf der Lebenszufriedenheit und des Wohlbefindens auf - mithin wichtigen Maßen des subjektiven Aspektes von seelischer Gesundheit (Stone et al. 2010). In der Analyse von sieben großen Datensätzen (Office for National Statistics Data für Großbritannien, BRFSS und GSS Data für die USA, Eurobarometer und ESS für Europa, ISSP Data für 45 Länder in sechs Erdteilen, Latino Barometer Data für Mittel- und Südamerika), gewonnen von über 1,3 Mio. TeilnehmerInnen aus der ganzen Welt, fanden Blanchflower und Oswald (2017) in den meisten Populationen eine konstante Abnahme der Lebenszufriedenheit bis hin zum Tiefpunkt zu Beginn der sechsten Lebensdekade, bevor dieses Maß bis zur achten Dekade wieder markant anstieg - auch dann, wenn der Einfluss verschiedener intermittierender Faktoren (z. B. Geschlecht, Bildungsstand, Erwerbsstatus, objektiver Gesundheitsstatus) statistisch kontrolliert wird.

Neben den subjektiven gibt es aber auch eine Reihe objektiver gesundheitlicher Veränderungen, die im Erwachsenenalter meist ab dem Beginn der vierten Lebensdekade stattfinden. Hierzu gehören das allmähliche Nachlassen der Seh- und Hörkraft, der Fertilität, der Verlust an Muskelmasse in der sechsten Lebensdekade sowie der Knochenstabilität. Geschlechtsunterschiede sind für viele dieser Rückgänge evident und Zusammenhänge mit hormonellen Regulationen nachgewiesen. Eine Liste dieser biologisch begründeten objektiven gesundheitlichen Veränderungen findet sich beispielsweise bei Riggs et al. (2008) oder Santrock (2015).

Interessanterweise bildete die Psychologie - hier vor allem die Persönlichkeits- und Entwicklungspsychologie - im Vergleich zu den beiden vorgenannten Disziplinen über viele Jahrzehnte einen gewissen Kontrast in der Betrachtung des jungen und mittleren Erwachsenenalters. Lange Zeit wurde diese Altersspanne als eine Lebensphase betrachtet, die vor allem durch hohe Stabilität individueller Merkmale charakterisiert sei: Es galt das auf William James (1842-1910) zurückgehende Verdikt, die individuelle Persönlichkeit sei mit dem Erreichen der Volljährigkeit quasi „set like plaster“, und es seien kaum noch relevante Veränderungen in Persönlichkeitsunterschieden zu verzeichnen, weder in der Kernpersönlichkeit (wie z. B. Extraversion oder Neurotizismus; Costa und McCrae 1994) noch in anderen gesundheitsbezüglichen Dispositionen des Einzelnen (wie z. B. seelische Gesundheit, Selbstwertgefühl, Depressivität; Becker 2006). 
Seit etwa drei Jahrzehnten wird nun aber auch in der Psychologie die Entwicklungsdynamik des jungen und mittleren Erwachsenenalters als eigenständiger Forschungsgegenstand entdeckt und das "Stabilitäts-Verdikt" hinterfragt (Baltes 1987; Lachman et al. 2015; Roberts und DelVecchio 2000; Specht et al. 2014; Van Dulmen 2013). Insbesondere biografische Transitionen haben nach aktuellem Forschungsstand das Potenzial, auf Persönlichkeitseigenschaften der Individuen differenziell einzuwirken, d. h. auf unterschiedliche psychische Bereiche bzw. Individuen je unterschiedlich. So berichten beispielsweise US-amerikanische Frauen, die zwischen 27 und 43 Jahren einen beruflichen Aufstieg erlebten, durchaus über Persönlichkeitsveränderungen wie etwa eine steigende Selbstwirksamkeit (die Autoren sprechen von agency) und gestiegene Normaffinität (norm adherence) im Vergleich mit Frauen, die derartige Aufstiege nicht erlebt hatten (Roberts 1997). Neyer und Asendorpf (2001) zeigten an einer studentischen Stichprobe, dass diejenigen Teilnehmer, die im Laufe von acht Jahren den Übergang vom Singleleben in eine feste Partnerschaft durchlebten, ihre emotionale Stabilität signifikant erhöhten - unabhängig davon, ob diese Partnerschaft aufrechterhalten wurde oder nicht. In solchen dynamischen Biografie-Persönlichkeits-Transaktionen scheint der Grad der Normativität (d. h. Erwartbarkeit) eines Übergangs ein bedeutsamer Faktor zu sein (siehe für eine Übersicht: Neyer et al. 2014). Durch solche und ähnliche Studien ist nun auch die Frage nach dem Zusammenhang zwischen sozialen Beziehungen, gesundheitsrelevanten Persönlichkeitsmerkmalen und dem individuellen Lebenslauf zu einem Forschungsthema der Psychologie geworden (Klauer und Greve 2005; Knoll und Schwarzer 2005; Weber 2005).

\subsection{Relevante Forschungsparadigmen zu Netzwerken und gesundheitlichen Ungleichheiten im jungen und mittleren Erwachsenenalter}

Der im Folgenden gegebene Überblick über den Forschungsstand zu sozialer Ungleichheit, Netzwerken und Gesundheit im jungen und mittleren Erwachsenenalter wird durch fünf übergreifende und interdisziplinäre Forschungsparadigmen gerahmt. Das erste, ursprünglich soziologische Paradigma des Lebenslaufs (1) (Mayer 2000; in der Psychologie häufig als Psychologie der Lebensspanne bezeichnet: Antonucci et al. 2010; Baltes und Smith 2004) betrachtet soziologische wie psychologische Phänomene in einem direkten konstitutiven Zusammenhang mit dem biologischen Alter, mit der Altersspanne, in der sich Individuen oder Bevölkerungsgruppen befinden, und mit der Sequenz bereits absolvierter bzw. zu absolvierender Übergänge. Das meint, dass 
das Lebensalter und die bisher erlebten Lebensübergänge hier nicht nur als Konkomitanten (begleitende Bedingungen), sondern als direkte Einflussfaktoren auf interessierende Phänomene mit betrachtet werden.

So beschreibt etwa Dragano (2007) die individuelle Abfolge biografischer Transitionen als einen zentralen Bestandteil der individuellen Belastungsbiografie. Diese stellt den biografischen Verlauf der Einwirkung patho- wie salutogener Faktoren dar, zu denen auch Netzwerkveränderungen gehören. In der Psychologie wird ein ähnlicher Ansatz im Ansatz der Kritischen Lebensereignisse verfolgt, in dem direkte und indirekte Belastungswirkungen nach dem Eintreffen unerwarteter oder unerwünschter Übergänge oder Widerfahrnisse erforscht werden (Klauer und Greve 2005). Gleichzeitig nimmt die Bedeutung individueller Bewältigungspotenziale für die Unterschiede in der Gesundheit mit steigendem Lebensalter zu, was die Notwendigkeit der Lebensspannenperspektive für Gesundheit und Netzwerke belegt (Lohaus und Klein-Heßling 2009; Wurm und Tesch-Römer 2005).

Weitere zentrale Paradigmen für dieses Kapitel beschreiben soziale Veränderungen in dieser Altersspanne, wobei entweder die sozialen Beziehungen (2) oder die sozialen Ungleichheiten (3) im Vordergrund stehen. Die meisten Forschungsarbeiten zur Veränderung von sozialen Beziehungen im jungen und mittleren Erwachsenenalter beziehen sich auf die spezifischen Veränderungen in einzelnen Beziehungsdomänen (soziale Bereiche), weniger auf die Veränderung von Netzwerken insgesamt (eine wichtige Ausnahme: Wrzus et al. 2013). Die Metaanalyse von Wrzus et al. (2013) über 243 Primärstudien, die meist im jungen und mittleren Erwachsenenalter die Größe des persönlichen Netzwerks untersuchten, zeigte einen Höhepunkt um das 30. Lebensjahr, zu dem sowohl Netzwerkgröße als auch der relative Anteil nicht-verwandter Personen am ausgeprägtesten sind, um danach kontinuierlich abzunehmen. Darüber hinaus ist zum Lebensspannenverlauf der Unterstützungs- und anderer Netzwerkeffekte im Hinblick auf Gesundheit (siehe Kap. ,Wirkmechanismen in sozialen Netzwerken“) wenig bekannt. Es ist bislang lediglich erforscht, dass Veränderungen in proximalen (d. h. engen, vertrauten) oder intimen Beziehungen (z. B. Liebesbeziehungen) immer mit systematischen Änderungen in den distalen Beziehungen (z. B. Freundes- und Bekanntennetzwerken) korrespondieren, etwa wenn die Auflösung einer Ehe mit dem Verlust des Kontaktes zu solchen Beziehungen des Ex-Partners einhergehen. Inwieweit sich soziale Beziehungen und soziale Ungleichheiten auch in gesundheitliche Ungleichheiten (4) übersetzen, wird in den folgenden Kapiteln erörtert.

Aus der Lebenslauf- oder Lebensspannenforschung ist weiterhin bekannt, dass sich das junge Erwachsenenalter als eine Hochphase des Ausprobierens verschiedener Gesundheits- und Risikoverhaltensweisen (5), aber auch als ein „Weichen-Alter“ für die Konsolidierung längerfristiger Gewohnheiten 
beschreiben lässt (Fooken und Kavšek 2012), deren Ausprägung eng an das erfolgreiche Erledigen phasenspezifischer Entwicklungsaufgaben und Transitionen gebunden ist. Unter Gesundheitsverhaltensweisen werden hierbei Handlungen verstanden, deren gesundheitsförderliche Effekte bekannt sind, wie z. B. eine ausgewogene Ernährung oder regelmäßiger Sport. Das Gegenteil davon sind Risikoverhaltensweisen, wie z. B. Tabak- oder Alkoholkonsum (Knoll et al. 2011). So diskutieren z. B. Sussman et al. (2011), dass gerade das junge Erwachsenenalter ein Risikoalter für gewohnheitsmäßigen Substanzkonsum darstellt und sich Ähnliches auch für andere Risiko- und Gesundheitsverhaltensweisen beobachten lässt. Somit wird gerade das junge Erwachsenenalter aus präventionsorientierter, gesundheitssoziologischer und psychologischer Sicht immer relevanter.

Für das Ende des jungen und mit Beginn des mittleren Erwachsenenalters (d. h. die Jahre um das 40. Lebensjahr herum) lässt sich in der gesundheitspsychologischen und sozialepidemiologischen Literatur ein weiterer Umschwung im individuellen Gesundheitsverhalten beobachten, der in einem markanten Anstieg im subjektiven Interesse an Gesundheits- und eine Aufgabe vieler wenngleich nicht aller - Risikoverhaltensweisen findet (Lachman et al. 2015). Dies wird auf die subjektive und sozial geteilte Wahrnehmung vieler Menschen in dieser Altersphase zurückgeführt, dass die Hochphase der physischen Kraft und Belastbarkeit an ein Ende gelangt und dass für das Bewahren der privaten wie beruflichen Leistungsfähigkeit ein neuer Fokus auf Gesundheit und Wohlbefinden notwendig ist.

Wir werden nun nach einem kurzen Aufgreifen einiger relevanter Theorien den jeweiligen Forschungsstand, das bisherige Wissen über die Zusammenhänge zwischen sozialer und gesundheitlicher Ungleichheit sowie sozialen Netzwerken und Gesundheit referieren. Dabei beziehen wir uns auf die genannten exemplarischen Transitionen des jungen und mittleren Erwachsenenalters als Motoren individueller Entwicklung und gesundheitlicher Ungleichheit.

\section{Spezielle psychologische Theorien der Lebenslauf- und Netzwerkentwicklung im Erwachsenenalter}

Im vorderen Teil dieses Bandes haben die Kapitel „Wirkmechanismen“ (siehe Kap. „Wirkmechanismen in sozialen Netzwerken“) und „Theorien“ (siehe Kap. „Soziale Beziehungen, soziales Kapital und soziale Netzwerke“) bereits wichtige theoretische Modelle vorgestellt, die hier nicht noch einmal wiederholt 
werden sollen. Wir möchten an dieser Stelle einige zusätzliche entwicklungs- und sozialpsychologische Theorien aus der Lebensspannenforschung heranziehen, da diese geeignet sind, den Zusammenhang von Netzwerkentwicklung und biografischen Transition zu plausibilisieren. ${ }^{1}$

Zwei sozial- und entwicklungspsychologische Theorien der Lebensspanne, die einen Zusammenhang von Transition und Beziehungsnetzen (und indirekt auch mit seelischer Gesundheit) im jungen und mittleren Erwachsenenalter beschreiben, sind einerseits die Theorie der Sozioemotionalen Selektivität (SST; Carstensen et al. 1999) und andererseits die Konvoi-Theorie nach Kahn und Antonucci (KT; Kahn und Antonucci 1980; Antonucci et al. 2011). Die Sozioemotionale Selektivitätstheorie geht von grundlegenden Entwicklungsaufgaben (Havighurst 1976) des Individuums aus, die eine Blaupause für die Konstruktion seiner sozialen Beziehungen abgeben. Entwicklungsaufgaben werden der Theorie zufolge durch das Individuum in Abhängigkeit von der subjektiv wahrgenommenen, verbleibenden Lebenszeit gewählt (,selegiert“). Während zu Beginn des Erwachsenenalters aufgrund einer subjektiv relativ „unbegrenzten“ Zukunftsperspektive vor allem Informationen gesammelt werden und Wirksamkeitsziele im Vordergrund stehen (Yeung et al. 2008), werden mit abnehmender subjektiver Lebenszeit Aufgaben der Emotionsregulation immer bedeutsamer. Bindungsmotive stehen dann im Vordergrund. Damit einhergehend werden der Theorie zufolge im jungen Erwachsenenalter tendenziell größere und loser gestrickte Netzwerke konstruiert, während im mittleren Erwachsenenalter persönliche Beziehungsnetze vermehrt derart umgestaltet werden, dass die proximalen (Familien-)Beziehungen intensiviert und distalere, z. B. kürzer bestehende und weniger vertraute Beziehungen, aufgegeben oder reduziert werden.

\footnotetext{
${ }^{1}$ Hier sei noch einmal auf die Abgrenzung von „Netzwerkeffekten“ zur bekannten „SocialSupport-Forschung“ hingewiesen. Während der enge Zusammenhang von wahrgenommener und geleisteter sozialer Unterstützung mit Gesundheit als allgemein bestätigt gelten kann (siehe z. B. die klassischen Meta-Analysen von Schwarzer und Leppin 1989; Smith et al. 1994), wird in der Netzwerkanalyse eine detaillierte Betrachtung der Zusammensetzung und Struktur von Beziehungsgesamtheiten verfolgt. In unsere Literaturrecherche wurden daher nur solche Studien ausgewählt, die konkrete Beziehungen zwischen Individuum und Netzwerkpartnern oder zumindest eine Gewichtung unterschiedlicher Netzwerksektoren (z. B. Familien- vs. Freundeskreise) berichteten. Erst ab dieser Tiefe in der Darstellung werten wir eine Studie als „Netzwerkstudie“ und beziehen sie in die Darstellung ein.
} 
Die Konvoi-Theorie geht wie die SST davon aus, dass Menschen nicht allein durch das Leben gehen, sondern sich fortwährend in einem relevanten Umfeld sozialer Beziehungen bewegen (engl. convoy $=$ Geleitzug). Dieser Convoy of Life gliedert sich der Theorie zufolge in proximale, eher stabile sowie distale, weniger stabile Beziehungen. Dabei konzipieren Antonucci et al. (2011) weniger die Veränderung der subjektiven Gewichtung beider Beziehungsarten über die Lebensspanne, sondern vielmehr eine fortwährende Relevanz beider. Der Unterschied besteht laut dieser Theorie vor allem darin, dass die distalen Beziehungen eher den Lebensumständen nach einem Übergang entsprechend umgebaut und angepasst werden müssen (z. B. indem nach einer Trennung oder einem Umzug neue Freundschaften eingegangen und alte de-intensiviert oder aufgegeben werden), während die proximalen (Kern-)Beziehungen des Netzwerks einen von diversen Transitionen relativ unabhängigen Bestand haben sollten.

Beide Theorien postulieren somit, dass proximale Beziehungen zu Familienmitgliedern und Freunden eher konstant über die Lebensspanne bleiben, während distale Beziehungen mit zunehmendem Alter aus intra-psychischen Gründen abnehmen (SST) bzw. von Lebensereignissen beeinflusst werden (KT). In einer Meta-Analyse zu beiden Ansätzen und der Veränderung der Netzwerkgröße über die Lebensspanne schließen Wrzus et al. (2013), dass sich empirisch Belege für beide Theorien finden lassen, sodass sich die Theorien eher in den zugrunde liegenden Mechanismen als in den Voraussagen zur Größe und Art der Netzwerke unterscheiden. Gleichwohl bleiben beide Netzwerktheorien bezüglich der Entwicklung von gesundheitlicher oder sozialer Ungleichheit defizitär, d. h. sie treffen keine unmittelbaren Vorhersagen hierzu.

Weitere Theorien der Netzwerkentwicklung im Erwachsenenalter sind eher struktureller Art oder fokussieren auf einzelne Domänen (soziale Bereiche). Die strukturorientierte Theorie der unterbrochenen Dyaden (dyadic withdrawal, Johnson und Leslie 1982) beispielsweise nimmt an, dass mit dem Übergang zur Partnerschaft, erst recht der Heirat, Freundesnetzwerke beider PartnerInnen kleiner werden. Hierbei kann es sein, dass verschiedene Dyaden, etwa die Partnerschaft und Freundschaftsbeziehungen, um die Ressourcen der Individuen konkurrieren (competition principle), womit die wachsende Zuwendung zu PartnerInnen oder Kindern Abstriche in den peripheren Beziehungen nach sich zieht. Neben der Konkurrenz sind im Sinne der Heider'schen Balancetheorie (siehe Kap. „Netzwerktheorie(n)“) jedoch auch ausgleichende bzw. harmonisierende Interaktionen von proximalen und distalen Beziehungen denkbar. Mit der Verkleinerung der individuellen Netzwerke gehen dann oft eine Homogenisierung und eine Überlappung der Freundesnetzwerke beider Partner einher (Kalmijn 2003), womit die Konkurrenz bzw. die Aufgabe von Freundesbeziehungen für 
eine Partnerschaft durch Balancierung - etwa die Konstruktion gemeinsamer Netzwerke - aufgefangen werden kann. Derartig verbundene Netzwerke (joint networks) gehören dann zum sozialen Kapital einer Partnerschaft oder Familie. Diese Form des sozialen Kapitals stabilisiert Paarbeziehungen und vertieft auch die Abhängigkeit der PartnerInnen voneinander (Kalmijn und Bernasco 2001). Über diesen Mechanismus können partnerschaftliche Netzwerke, ebenso wie der berufliche Erfolg, Persönlichkeitsmerkmale wie die bereits erwähnte Normaffinität (norm adherence) der PartnerInnen verstärken (Milardo und Allan 2000).

Die Theorie der Beziehungsturbulenz, (relational turbulence, Solomon und Knobloch 2004) zielt darauf ab, dass Transitionen und die mit ihnen einhergehenden Netzwerkveränderungen nicht ohne Auswirkung auf die proximalen Beziehungen - vor allem die Partnerschaft - bleiben. Viele Transitionen oder Ereignisse im Erwachsenenalter können Partnerbeziehungen gefährden, seien es berufliche Schwierigkeiten, ungewollte Kinderlosigkeit oder schwere Erkrankungen (Nagy und Theiss 2013). Derartige Herausforderungen an die Partnerschaft werden dann oft mit der Änderung von Beziehungsskripten (Normen und Routinen) bewältigt, die adaptiv (z. B. gesundheitsförderlich), aber auch maladaptiv (z. B. gesundheitsgefährdend) sein können. Mit der Theorie der Kritischen Lebensereignisse (KLE; Filipp und Aymanns 1987) lässt es sich genauer beschreiben, dass eine zentrale Aufgabe des Erwachsenenalters in der Bewältigung derartiger Turbulenzen und der Aufrechterhaltung einer relativen Stabilität in der Partnerschaft besteht. In der gesundheitswissenschaftlichen und sozialepidemiologischen Lebenslaufforschung wird gesehen, dass sich unbewältigte kritische Lebensereignisse langfristig negativ auf die Gesundheit auswirken können (Steptoe 1998, zit.n. Marmot 2000). Auch diese Theorien sind eher psychologischer Natur, d. h. es fehlt ihnen an Einbettung in den Kontext der Entwicklung gesundheitlicher Ungleichheit. Kritische Lebensereignisse allerdings variieren in Häufigkeit und Schweregrad in Abhängigkeit von der sozialen Herkunft.

Ausgehend von den genannten paradigmatischen Begriffen stehen für uns im Folgenden diejenigen biografischen Übergänge im Vordergrund, an denen die Veränderungen sozialer und gesundheitlicher Ungleichheiten sowie von Netzwerken im Erwachsenenalter illustriert und diskutiert werden können. Exemplarisch werden dafür der Übergang zur Partnerschaft/Heirat, die Scheidung/ Trennung und der Auszug der eigenen Kinder aus dem Elternhaus herausgegriffen. Diese Auswahl wird dadurch begründet, dass einerseits das Wechselspiel soziologischer und psychologischer Effekte gut verdeutlicht werden kann und dass diese Transitionen andererseits erhebliche Auswirkungen auf das Leben und die Gesundheit im Erwachsenenalter haben können. Andere relevante Transitionen des Erwachsenenalters, wie der Übergang in die Erwerbslosigkeit 
(siehe Kap. „Arbeitslosigkeit, soziale Netzwerke und gesundheitliche Ungleichheit") werden an anderen Stellen dieses Bandes behandelt. Während das Eingehen einer Partnerschaft und der Auszug der Kinder normative Transitionen darstellen, die für die Mehrheit der Erwachsenen erwartbar sind, bleibt Scheidung trotz relativ hoher Prävalenzen ein nicht-normatives Ereignis. Damit ist gemeint, dass es sich nicht - anders als die beiden erstgenannten - um einen Übergang handelt, der im individuellen Lebenslauf als fest erwartbar und erwünscht betrachtet werden kann, sondern zumeist als krisenhafte Widerfahrnis und aversiv erlebt wird (Filipp und Aymanns 1987).

\section{Ungleichheit (SES) und Gesundheit}

Der enge Zusammenhang zwischen sozialer und gesundheitlicher Ungleichheit im Erwachsenenalter ist in zahlreichen sozialepidemiologischen Studien aufgezeigt worden (für einen Überblick siehe z. B. Cutler und Lleras-Muney 2010; Hurrelmann und Richter 2013; Mielck 2005). So rauchen bildungsfernere Personen beispielsweise mehr, ernähren sich ungesünder und sterben früher als bildungsnähere (Hoffmann et al. 2018). Es gibt insgesamt eine besonders große gesundheitliche Belastung der unteren Statusgruppen, wobei dieser Effekt interessanterweise in einigen eher egalitären Gesellschaften wie den skandinavischen abgemildert scheint. In einer epidemiologischen Studie mit 1003 norwegischen Personen im mittleren Erwachsenenalter (46,3 Jahre) fanden Dalgard und Håheim (1998) beispielsweise, dass Einkommen keinen signifikanten Einfluss auf die Sterblichkeit im Verlauf von 17 Jahren hatte.

Für den deutschen Epidemiologen Mielck (2005) beispielsweise wird der für viele Länder typische, enge Zusammenhang zwischen sozialer und gesundheitlicher Ungleichheit dadurch erklärt, dass statusniedrige gesellschaftliche Gruppen deshalb weniger gesund sind als statushöhere, weil sie mehr Risiko- und weniger Gesundheitsverhalten zeigen. Zudem seien diese Gruppen statusbedingt mehr biografischen Belastungen (z. B. materiellen Entbehrungen, schlechteren Arbeitsbedingungen) ausgesetzt, erhielten weniger oder ineffektivere soziale Unterstützung und können oft weniger gut von der medizinischen Versorgung oder Vorsorge profitieren. Mit diesen Mechanismen wären etliche der in Abschn. 1.2 bezeichneten Zusammenhänge erklärt. Übertragen auf die biografischen Übergänge während des jungen und mittleren Erwachsenenalters würde dies bedeuten: Statusniedrige Personen erleben in dieser Alterspanne mehr Belastungen, u. a. mehr belastende Transitionen, erhalten bei diesen weniger oder ineffektivere soziale Unterstützung und können zudem bei der Bewältigung wenig gut als 
statushöhere Gruppen von professioneller (z. B. medizinisch-psychologischer) Gesundheitsfürsorge profitieren. Welche Belege lassen sich in der Literatur für diese Annahmen finden?

\section{1 Übergänge in Partnerschaften als Motor von gesundheitlichen Ungleichheiten}

Das vorherrschende Muster des Eingehens und Lösens von Partnerschaften junger Erwachsener in westlichen Industriegesellschaften ist als sequenzielle Monogamie bezeichnet worden (z. B. Morris und Kretzschmar 1995). Das bedeutet, dass sich zumeist mit einem Partner oder einer Partnerin verbunden wird, dabei aber mehrere Partnerschaften unterschiedlicher Dauer aufeinander folgen. Hier lassen sich deutliche interindividuelle Unterschiede darin finden, wann und $o b$ geheiratet oder ob eher unverheiratet als Paar zusammengelebt wird und wie viele Partnerschaften in dieser Altersspanne insgesamt eingegangen werden (Schneider und Rüger 2008). Beide Parameter hängen sowohl mit Ungleichheitsdimensionen wie auch mit Gesundheit zusammen.

Als empirisch gesichert gilt, dass stabile Partnerschaften positive Einflüsse auf das subjektive Wohlbefinden haben (Schütz und Wiesner 2000). Auch für körperliche Erkrankungen stellen sie einen häufig bestätigten Schutzfaktor dar, was insbesondere für Männer zu gelten scheint (healthy-marriage-hypothesis, Carr und Springer 2010; Lillard und Waite 1995; Dalgard und Håheim 1998; Soons et al. 2009). Bei Frauen sind Unterschiede im sexuellen Risikoverhalten zwischen ledigen und verheirateten Personen gefunden worden (Wayment et al. 2003). Somit lässt sich das diesem Abschnitt zugrunde liegende Thema in die Frage übertragen, inwieweit die Stabilität von Partnerschaften geeignet ist, zwischen sozialen und gesundheitlichen Ungleichheiten zu vermitteln.

In der aktuellen Forschung werden signifikante, aber numerisch nicht allzu große Unterschiede im Partnerschaftsverhalten der Bildungsschichten beschrieben: Statusniedrigere Personen heiraten früher als statushöhere, dafür sind ihre Ehen weniger stabil (Schwartz und Han 2014). Dieser Befund ist vor dem Hintergrund unserer Fragestellung dahin gehend interessant, dass häufiger wechselnde Partner als ein Risikofaktor für sexuell übertragbare Erkrankungen beschrieben werden (Millstein et al. 1994). Beide Prozesse (Stabilität und Wechsel) wären demnach geeignet, zu gesundheitlichen Ungleichheiten beizutragen, sofern sich ihre soziale Schichtung weiter belegen ließ. Hierzu gibt es unseres Wissens jedoch keine einheitliche Befundlage (vgl. etwa die widersprüchlichen Ergebnisse bei Kupek 2001; Rissel et al. 2014; Tanfer et al. 1995). 
Trennungen oder Scheidungen hingegen ${ }^{2}$ werden in der Literatur eindeutiger als Gesundheitsrisiko betrachtet, das sich insbesondere durch eine Zunahme von Risikoverhaltensweisen (z. B. Alkohol, Promiskuität) sowie von psychischer Belastung (z. B. geringeres Wohlbefinden, Zunahme von Depressivität) gesundheitlich auswirken kann (Leopold 2018). Dass eine Scheidung insbesondere dann zu psychologischem Stress und Anpassungsschwierigkeiten führt, wenn wahrgenommene ökonomische Ressourcen für ihre Bewältigung unzureichend sind, kann eine jüngere iranische Studie mit 800 geschiedenen Frauen (Durchschnittalter 38,8 Jahre, 1 - 2 Jahre nach der formalen Scheidung) zeigen (Esmaeili et al. 2015). In den Ergebnissen der querschnittlichen Regressionsanalyse erklärten Bildungsjahre (beta $=-.05$ ), Kinderzahl $($ beta $=.45)$ und geringer ökonomischer Status (beta $=.46)$ multivariat $63.6 \%$ der Varianz in der psychischen Symptomatik der Frauen. Hier gehen soziale und gesundheitliche Ungleichheiten Hand in Hand.

\subsection{Empty nest}

Einen weiteren biografischen Übergang im Erwachsenenalter stellt der Auszug der Kinder aus dem Elternhaus dar. Hierfür hat sich in der Forschung der Begriff der „empty nest-Situation“ eingebürgert ${ }^{3}$. Es ist schon längere Zeit bekannt, dass die Mehrheit der Eltern diesen Übergang ohne schwerwiegende emotionale, gesundheitliche oder ökonomische Probleme bewältigt (Harkins 1978), was auch ein Effekt des historisch veränderten Kindernutzens ist. Kinder, die in industrialisierten Wohlfahrtsgesellschaften das Elternhaus verlassen, entziehen diesem kaum noch ihre Arbeitskraft, und emotionale Nähe lässt sich auch nach dem Auszug herstellen - oft sogar besser als vorher (Papastefanu 2000).

Soziale Unterschiede werden deshalb nur dort mit dem Auszug der Kinder assoziiert, wo die Kinder mehr als emotionalen Nutzen für die Familie haben.

\footnotetext{
${ }^{2}$ Wir machen im Folgenden keinen Unterschied zwischen der Trennung verheirateter oder unverheirateter Paare oder zwischen dem Übergang zur faktischen Trennung vs. der rechtlichen Scheidung eines verheirateten Paares, und nutzen der Einfachheit halber nur den Begriff der Scheidung.

${ }^{3}$ In der Lebenslaufforschung herrscht Uneinigkeit, wie diese Transition korrekt benannt werden sollte. Einerseits ist das Familiennest nach dem Auszug der Kinder nicht „leer“, andererseits hören auch die Eltern mit dem Auszug ihrer Kinder nicht auf, Eltern zu sein (postparental period). Für eine Übersicht siehe Bouchard (2014).
} 
Dies betrifft insbesondere traditionelle und rurale Gesellschaften und ärmere soziale Schichten in industrialisierten Gesellschaften. Besondere Relevanz hat die empty nest-Situation daher beispielsweise in China, wo sie auch breit diskutiert wird (Wan et al. 2008). Dort sind Kinder nach wie vor eine wesentliche Quelle der Unterstützung Älterer und damit ihrer Gesundheit. Als Folge der jahrzehntelang verfolgten Ein-Kind-Politik und kaum vorhandener Wohlfahrt müssen zwei erwerbstätige Erwachsene die Kosten für vier Ältere und ein Kind erwirtschaften (eine sogenannte 4-2-1-Familienkonstellation; Wan et al. 2008). Darüber hinaus macht die massenhafte Abwanderung erwachsener Kinder in die Städte vor allem das Alter auf dem Lande zu einem gesundheitlichen Hochrisiko (Liu und Guo 2008).

In stärker industrialisierten Ländern hat sich hingegen der Zeitpunkt des Auszuges nicht nur nach hinten verlagert (Beaupré et al. 2006); es wird auch der Wiedereinzug erwachsener Kinder zunehmend diskutiert. In „crowded nests“ wohnen erwachsene Kinder neben ihren Eltern, wenn die Kinder beispielsweise aufgrund abnehmender Reallöhne oder zyklischer Wirtschaftskrisen nicht genug Ressourcen haben, einen eigenen Haushalt zu gründen. Hier treffen die „Boomers“ auf die „Boomerangers“" . Für Europa liegen hierzu nur wenige Daten vor, die jedoch zeigen, dass es neben kulturellen auch sozioökonomische Faktoren zu geben scheint, die einen Wiedereinzug erwachsener Kinder in das Elternhaus und verlängerten Elternnutzen wahrscheinlicher machen (Kleinepier et al. 2017). Sowohl Auszüge als auch Gründungen eigener Haushalte werden in Industriegesellschaften mit hoher Jugendarbeitslosigkeit und/oder hohen Heiratshürden zunehmend problematisch (Mínguez 2016; South und Lei 2015). Das Zusammenleben mit erwachsenen Kindern scheint der Lebenszufriedenheit wiederum eher abträglich zu sein und einen relevanten psychischen Stressor darzustellen (Pollmann-Schult 2011). Hier soll nur festgehalten werden, dass sozioökonomischer Wandel und finanzielle Ressourcen das Timing und die Art der Übergänge nachhaltig beeinflussen. Damit wird ein weiterer Mechanismus deutlich, über den sich soziale auf die gesundheitliche Ungleichheit auswirken kann.

\footnotetext{
${ }^{4}$ Dieses im angelsächsischen Sprachraum gebräuchliche Wortspiel meint, dass die Eltern aus der Baby-Boomer-Generation (in Deutschland die Mitte der 1950er bis Ende der 1960er Jahre geborenen Personen) ihre Kinder zunehmend häufiger und länger im elterlichen Haushalt behalten, oft als „Rückkehrer“ (boomerang) nach fehlgeschlagenen Auszugsversuchen.
} 


\section{$4 \quad$ Netzwerke und Gesundheit}

\subsection{Partnerschaftsübergänge als Motor von Netzwerkeffekten auf Gesundheit}

Weder das Eingehen noch das Auflösen von Partnerschaft findet außerhalb sozialer Kontexte oder auf einer „Insel“ statt. Diese Metapher wird in den grundlegenden Arbeiten von Felmlee (2001) und Sprecher et al. (2006) zur Bedeutung sozialer Netzwerke für Partnerschaften herangezogen. Die Autoren beschreiben mindestens drei grundlegende Mechanismen, über die soziale Beziehungsnetze auf partnerschaftliche Übergänge einwirken können. Zum einen stellen Netzwerke Möglichkeiten für das Kennenlernen von potenziellen Partnern dar und bestimmen somit durch ihre Zusammensetzung sowie Struktur die Wahrscheinlichkeit für das Eingehen einer Partnerschaft oder sexuellen Beziehung. Zum zweiten treffen Individuen in ihren Netzwerken stets auch auf unterschiedliche Ausmaße an Anerkennung vs. Ablehnung ihres partnerschaftlichen Handelns (z. B. Partnerwahl, Heirat, Trennung), wodurch der fragliche Übergang für das Individuum in unterschiedlichem Ausmaß auch wahrscheinlicher bzw. sozial unterstützt wird. Drittens stellen Netzwerke immer auch mehr oder weniger attraktive Beziehungsalternativen zur Verfügung und ver(un)wahrscheinlichen damit partiell eine Trennung - was sich dann wiederum auf individuelle gesundheitliche Merkmale auswirken kann.

In ihrer Darstellung des diesbezüglichen Forschungsstandes schließen Sprecher et al. (2006), dass unterstützende (approving) soziale Netzwerke als förderlich für das Eingehen und die Stabilität von Paarbeziehungen angesehen werden können und dass umgekehrt eine Scheidung oder Trennung mit einer signifikanten Abnahme des Verwandten- und Verheirateten-Anteils im Netzwerk sowie der Zunahme des Kollegen- und Singleanteils einhergeht. Aber nicht nur die Zusammensetzung, auch die Struktur des Netzwerkes scheint mit Paarstabilität einherzugehen, wie eine Arbeit von Widmer et al. (2004) zeigt: Paare mit individualisierten (individualized) oder sich einmischenden (interfering) Netzwerken berichteten über eine signifikant geringere Paarzufriedenheit sowie stärkere Trennungsgedanken als Paare mit überlappenden (overlapping) Netzwerken. Erste Pilotstudien zu diesem wichtigen Zusammenhang von Netzwerk und Paarstabilität und -zufriedenheit finden sich bereits z. B. bei Milardo (1989), Levitt et al. (1986), Hansen et al. (1991) oder Burger und Milardo (1995). Die Studie von Veiel et al. (1991) zeigte beispielsweise, dass sowohl die Ähnlichkeit als auch die Überlappung des partnerschaftlichen Netzwerks für die Bewältigung belastender Anforderungen funktional sein kann. 
Aber auch jenseits des Stabilitätseffektes sozialer Netzwerke finden sich in der Literatur Hinweise auf die direkte gesundheitliche Bedeutung nach einer erfolgten Scheidung. Die frühe Längsschnittstudie von Hughes et al. (1993) befragte 29 alleinerziehende US-Amerikanerinnen drei sowie acht Monate nach der juristischen Scheidung zu ihren Netzwerken und ihrer psychischen Gesundheit. Im zeitlichen Umfeld der Scheidung wurde über eine hohe Dynamik in den Freundesbeziehungen berichtet: Bereits in den Monaten um den Zeitpunkt der Scheidung herum waren den Studienteilnehmerinnen im Schnitt knapp fünf Freunde verloren gegangen, bis zum zweiten Interview waren es noch einmal vier weitere Freunde. Gleichzeitig kamen in diesem Zeitraum circa fünf neue Freunde dazu. Während die Anzahl der Unterstützungsleister im Netzwerk beim ersten sowie zweiten Interview meist positiv mit der psychischen Gesundheit beim zweiten Interview zusammenhing, sagte ein hoher Eltern- und geringer Freundes- sowie Geschwisteranteil im Netzwerk eher Anpassungsschwierigkeiten und eine geringere seelische Gesundheit bei den alleinerziehenden Frauen voraus. Auch die Ergebnisse von Stone (2002) zeigen anhand der Zusammensetzungs- und Unterstützungsmaße der Netzwerke von 101 geschiedenen Vätern, dass seelische Gesundheit positiv mit den nach der Scheidung neu ins Netzwerk hinzugekommenen Vertrauten und ihrer Unterstützung zusammenhängt.

Einen weiteren Zusammenhang zwischen der Zusammensetzung der sozialen Netzwerke und depressiven Symptomen nach einer Scheidung berichten Kincaid und Caldwell (1991). In ihrer Analyse von 56 geschiedenen Personen aus der Milwaukee Family Study zeigte sich, dass insbesondere diejenigen Personen, die die Scheidung selbst nicht initiiert hatten, von einem höheren Verwandtenanteil im Netzwerk zu profitieren schienen, indem sie signifikant geringere Depressionswerte berichteten. Bei den scheidungsinitiierenden Personen war der Zusammenhang schwach gegenläufig im Sinne eher marginal höheren Depressivität bei höherem Verwandtenanteil im Netz.

Die Ergebnisse in Bezug auf die sozialen Netzwerke von Geschiedenen sowie ihre Gesundheitseffekte sind also weiter uneinheitlich, was in der Literatur durchaus gesehen und diskutiert wird (Ertel et al. 2009; Kalmijn et al. 2005). Hier liegt ein vielversprechendes Feld für zukünftige Forschung.

Einen einzigartigen Einblick in den Zusammenhang von partnerschaftlichen Netzwerken und seelischer Gesundheit liefert die Studie von Murphy et al. (1998). Hier ging es um die Bewältigung eines anderen und seltenen traumatischen Familienübergangs, nämlich des gewaltsamen Todes des eigenen Kindes in dessen Jugend- oder jungen Erwachsenenalter. Die Daten von 261 trauernden Elternteilen zeigen, dass sich vertrauensvolle und unterstützende Netzwerkbeziehungen tendenziell positiv im Sinne einer geringeren psychischen und physischen Symptomatik sowie einer höheren Partnerschaftszufriedenheit auswirkten. 


\subsection{Empty-Nest als Motor von Netzwerkeffekten auf Gesundheit}

Kinder spielen in den Netzwerken von Eltern eine nicht unerhebliche Rolle für deren Gesundheit, wenn auch nicht klar ist, in welcher Funktion. Eltern partizipieren stärker als Kinderlose an der Gesellschaft, ob bürgerschaftlich, politisch oder religiös. Dieser „Kinder-Effekt“ geht mit dem Auszug derselben aus dem Elternhaus größtenteils verloren (Pollmann-Schult 2011). Die Eltern-KindDyaden werden mit dem Auszug der Kinder aber meist nicht unterbrochen, sondern auf andere Kommunikationskanäle verschoben. US-amerikanische Eltern beispielsweise erhöhten ihre elektronische Kommunikation mit den Kindern, nachdem diese ausgezogen waren für etwa zwei Jahre (Tanis et al. 2017). Vorläufer depressiver Erkrankungen, wie Gefühle der Einsamkeit und des Verlassenseins, konnten so erfolgreich reduziert werden. In einer chinesischen Studie wurde gezeigt, dass diese elektronische Art der Kommunikation für Ältere weniger befriedigend ist als die direkte Interaktion (Sun et al. 2016) - was allerdings wiederum von der Erreichbarkeit der Kinder abhing. Sind Kinder räumlich für den direkten Kontakt erreichbar, wird die digitale Kommunikation als weniger befriedigend empfunden.

Im Hinblick auf das erweiterte Netzwerk konnte Kalmijn (2003) für die Niederlande zeigen, dass sowohl die Anzahl der Freunde von Eltern als auch die Intensität des Kontaktes mit ihnen am geringsten ist, wenn die Kinder ausziehen. Allerdings spielte für diesen Effekt wahrscheinlich auch das Alter der Befragten eine Rolle, das in dieser Untersuchung nicht kontrolliert wurde. Verglichen mit dem Gründen eines gemeinsamen Haushalts, der eine sprunghafte Verkleinerung der Freundesnetzwerke zur Folge hatte, war der Auszug der Kinder aus dem Elternhaus für das Netzwerk weniger bedeutend. Wird jedoch der Anteil gemeinsamer Freunde beider Eltern betrachtet, hat das leere Nest einen eigenständigen Effekt. Die über die Lebensspanne kontinuierlich zunehmende Verflechtung der Freundesnetzwerke beider Eltern erfährt eine sichtbare Steigerung, sobald die Kinder das Elternhaus verlassen. Dabei schöpfen die Eltern aus dem Pool der sogenannten asymmetrischen Freundschaften, das heißt, es werden insbesondere solche Freunde in das gemeinsame Netzwerk aufgenommen, die vom Partner vor der Transition als Freunde des anderen benannt wurden - aber nicht von den Betroffenen (Kalmijn 2003). Direkte Effekte auf Gesundheit oder Partnerschaftszufriedenheit wurden in dieser Studie nicht berichtet.

Ein Mechanismus, über den das leere Nest mit Netzwerken und Gesundheit wechselwirken kann, führt über die Beeinflussung der elterlichen Partnerschaft. Wenn es im Sinne des Turbulenzmodells (siehe Abschn. 2) nicht gelingt, 
die elterliche Beziehung für diese Phase gewissermaßen „,neu zu erfinden“, beispielsweise in Form gemeinsamer Zeit mit dem partnerschaftlichen Netzwerk, werden Konflikte und Trennungen wahrscheinlicher. Auch die allgemein mit Scheidungen verbundenen ökonomischen Risiken werden durch den Zeitpunkt der Trennung moderiert, da mit zunehmenden Alter die Wahrscheinlichkeiten für Neuorientierungen, sei es auf dem Partner- oder dem Arbeitsmarkt, geringer werden. In Partnerbeziehungen kann der Auszug der Kinder deshalb Ängste vor Abhängigkeit generieren, insbesondere bei Frauen (Nagy und Theiss 2013), die offenbar dann stärker wirken können, wenn das Netzwerk wenig unterstützt. Zum direkten Einfluss dieser Effekte auf objektive Gesundheitsmaße liegen bislang allerdings keine Studien vor.

\section{$5 \quad$ Ungleichheit, Netzwerke und Gesundheit}

Während es für den Zusammenhang von sozialer Ungleichheit, wahrgenommener sozialer Unterstützung und Gesundheit zumindest einige Studien gibt, sind Arbeiten mit eigentlichen Netzwerkparametern seltener. Wir müssen daher unseren Fokus auf Partnerschaftsübergänge und Empty-Nest-Situation im jungen und mittleren Erwachsenenalter an dieser Stelle aufgeben (mit Ausnahme einiger Scheidungsfolgestudien am Ende dieses Abschnitts), um dennoch einige Studien berichten zu können.

Insgesamt ist vorwegnehmend bereits festzustellen, dass die überwiegende Zahl der Studien zu den interessierenden Zusammenhängen methodisch entweder einen Moderator- oder einen Mediatoransatz verfolgt. Während Mediatoranalysen einen eigenständigen Zusammenhang von sozialen Ungleichheiten (im Folgenden häufig mit SES abgekürzt für socio-economic status) mit sozialen Netzwerken annehmen, so ist dies bei Moderatormodellen nicht notwendig. Abb. 1 stellt diese beiden unterschiedlichen Ansätze, die auch in vielen anderen Beiträgen dieses Bandes thematisiert werden, grafisch dar.

Einen Mediatoreffekt von sozialen Netzwerken auf den Zusammenhang zwischen sozialer und gesundheitlicher Ungleichheit fand die Studie von Rubin et al. (2016). Hier hatten 316 australische Studierende im ersten Semester zunächst ihren SES anhand einer Skala entlang von Geld, Bildung und Berufstätigkeit im Vergleich mit anderen Menschen in Australien einzuschätzen. Dann beurteilten sie ihre mentale Gesundheit mit der Depression Anxiety Stress Scale und einer angepassten Version der Satisfaction with Life Scale sowie ihr soziales Netzwerk anhand der Anzahl aktueller Freunde an der Universität und der Häufigkeit 

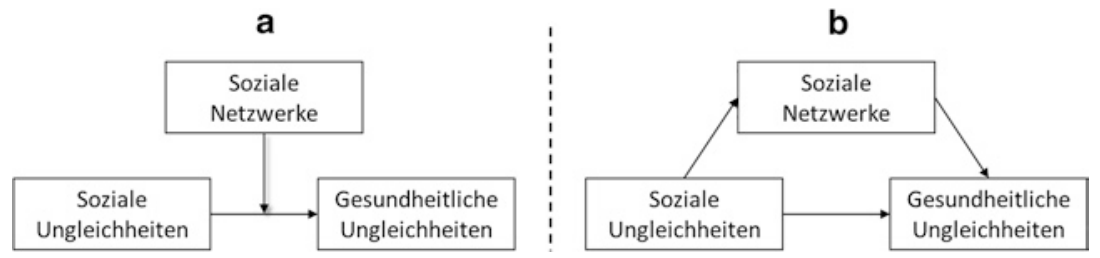

Abb. 1 Typische Designs von Moderator (a)- und Mediator (b)-Analysen zum Zusammenhang von SES, Netzwerken und Gesundheit. (Quelle: Eigene Darstellung)

der wöchentlichen Kommunikation mit diesen. Über einen kurzen Längsschnitt von einem Jahr fand sich zunächst der erwartete Effekt von initialem SES auf Gesundheitsmaße ein Jahr später, der partiell durch das individuelle Ausmaß sozialer Netzwerke mediiert wurde.

Cable et al. (2013) ermittelten dieselbe Mediation mithilfe von 3000 Teilnehmern der englischen NCDS-Studie. Als SES-Maß nutzte diese Studie die Bildung in Jahren. Für die Messung der Gesundheit wurde ein Mental Health-Inventar eingesetzt, während netzwerkseitig die Zahl der engen Familienmitglieder sowie die Zahl der Freunde erhoben wurde. Geringer gebildete Personen zeigten geringere Werte in seelischer Gesundheit und darüber hinaus größere Familienund kleinere Freundesnetzwerke. In der Mediationsanalyse konnten die Autoren einen eigenständigen Effekt dieser Netzwerkmaße auf die seelische Gesundheit zeigen: bei Männern wirkten sich größere Netzwerke (unabhängig vom Netzwerkbereich), bei Frauen lediglich Freundessektoren partiell mediierend auf den Zusammenhang von SES und Gesundheit aus.

Einen ähnlichen Mediationseffekt für soziale Netzwerke konnten Vonneilich et al. (2012) im Längsschnitt mit einer westdeutschen Stichprobe am Ende des mittleren Erwachsenenalters $(n=4146$ Teilnehmer; Mage=58,8 Jahre zum 2 . Zeitpunkt des 5-Jahres-Längsschnitts) quantitativ beziffern. Die prospektiven und signifikante Effekte von Bildung, Einkommen und Berufsstatus auf den subjektiven Gesundheitsstatus der Teilnehmer reduzierten sich durch die Hinzunahme eines zusammengesetzten Indexes für die soziale Einbettung (SII nach Berkman, bestehend aus drei Indizes für die Anzahl enger Beziehungen, die Einbindung in Vereine oder anderen Gruppen sowie den Familienstand) signifikant. Dabei beliefen sich diese signifikanten Reduktionen der direkten Effekte durch die Hinzunahme des SII (plus zwei weiterer Unterstützungsmaße) bei der Gesamtstichprobe auf 19,0 \% für den Effekt von Bildung auf Gesundheit, auf 21,1\% für 
Berufsstatus und auf 26,6 \% für Einkommen. Die Autoren schließen zusammenfassend, dass die Ergebnisse einen deutlichen Hinweis auf die mediierende Wirkung sozialer Netzwerke für den Einfluss sozialer Ungleichheits- auf Gesundheitsvariablen darstellen.

Chappell und Funk (2010) wiederum konnten in einer Studie mit 916 kanadischen Teilnehmern einer Disadvantage Study diese Mediation in Bezug auf allgemeine Gesundheitsmaße jedoch nicht finden. Ungleichheit wurde hier über Einkommen und Bildung gemessen, Gesundheit über die RAND Health Survey Scale und Netzwerke über die egozentrierte Netzgröße sowie die Anzahl sozialer Vereine, in denen sich die Teilnehmer engagierten. In der Analyse zeigte sich der erwartete Effekt von Einkommen auf den Health Status, Netzwerkmaße trugen jedoch an keiner Stelle zu den statistischen Modellen der Analyse signifikant bei (keine Mediation).

\subsection{Scheidung, SES, Netzwerke und Gesundheit}

Die Studie von Steptoe und Marmot (2003) mit den Whitehall-2-Daten (227 Teilnehmer zwischen 47 und 59 Jahre alt) untersuchte den möglichen Zusammenhang zwischen SES, Scheidung, Netzwerken und Gesundheit sowie Gesundheitsverhalten. Ein aus den Indikatoren Netzwerkgröße, emotionale Unterstützung, berufliche, finanzielle und nachbarschaftliche Belastungen sowie persönliche Bewältigungsstile zusammengesetzter psychosozialer Belastungsindikator (PAVIX; hohe Werte stehen für die jeweiligen Risikoausprägungen der Indikatoren, wobei eine geringe Netzwerkgröße als Risikoausprägung gewertet wird) korrelierte zunächst negativ mit SES. Ein geringerer SES ging mit höheren psychosozialen Belastungen einher - ganz wie bereits in Abschn. 3 anhand der Studien von Mielck und anderen beschrieben wurde. Auch zeigten sich - analog der bereits berichteten "healthy-marriage-hypothesis“ - verheiratete Personen hinsichtlich dieser Indikatoren signifikant unbelasteter als nicht verheiratete (darunter auch Geschiedene). In einem Regressionsmodell, das all diese Maße für die Erklärung gesundheitlicher Indikatoren (z. B. Depression, Schlafstörungen, Hoffnungslosigkeit, subjektives Gesundheitsgefühl etc.) nutzte, verblieb nur noch der PAVIX als signifikanter Prädiktor. Weder SES noch der Familienstand sagten die Gesundheitsmaße nach Einschluss des PAVIX vorher. Dieselben Ergebnisse zeigten sich auch bei physiologischen Gesundheitsmaßen, nicht aber beim Gesundheitsverhalten selbst. Diese Befunde ließen sich als eine vollständige Mediation der bekannten Gesundheitseffekten von SES und Familienstand durch soziale (Netzwerk-)Ressourcen interpretieren, was von den Autoren 
als ein Argument für die Stärkung dieser Aspekte in der zukünftigen Gesundheitsforschung und -praxis gewertet wurde.

In einer niederländischen Studie (Terhell et al. 2004) konnten die persönlichen Netzwerke von 104 geschiedenen Männern und Frauen über einen Zeitraum von insgesamt durchschnittlich 11,7 Jahren beobachtet werden (von 4,2 Monaten [T1] und 1,1 Jahren [T2] bis 12,1 Jahren [T3] nach der Scheidung). Zusätzlich wurde retrospektiv nach dem Jahr vor der Scheidung [T0] gefragt. Die Autoren unterschieden clusteranalytisch vier verschiedene Muster der Netzwerkveränderungen in ihrer Stichprobe: eine signifikante und dauerhafte Verkleinerung des Netzwerks über alle Zeitpunkte hinweg (38,5\% der Befragten) eine Verkleinerung des Netzwerks im Jahr nach der Scheidung mit nachfolgender Erholung fast auf das Ausgangsniveau (28,8 \%) eine kurzfristige Erhöhung der Netzwerkgröße im Jahr nach der Scheidung mit nachfolgendem Absinken unter das Ausgangsniveau $(14,4 \%)$ sowie eine signifikanten und dauerhafte Vergrößerung des Netzwerks über alle Zeitpunkte hinweg (15,4\%). Interessant an den Ergebnissen dieser Studie für unsere Frage ist, dass sich weder Bildung noch seelische Gesundheit (operationalisiert über den Selbstwert und emotionale Stabilität) signifikant zwischen diesen vier Mustern unterschieden, sondern lediglich Geschlecht, Alter und Scheidungsmerkmale signifikant zwischen den Mustern differenzierten.

$\mathrm{Zu}$ etwas anderen Ergebnissen gelangen Symoens et al. (2014) mit Daten des European Social Survey (ESS3, $n=18.376$ jemals verheiratete 25- bis 60-Jährige). Symoens und Kollegen finden zunächst die erwarteten Unterschiede in seelischer Gesundheit zwischen Geschiedenen und Verheirateten $(d=0.28$, $p<.001$ ), können aber zeigen, dass diese Unterschiede sich halbieren, wenn die „Netzwerkmaße“ in die Regressionen einbezogen werden. Bildungsjahre als Indikator für den SES haben bereits einen Haupteffekt auf Depressivität (keine Daten in der Primärstudie berichtet), machen aber auch unter Einbezug der „Netzwerkmaße“ einen signifikanten Unterschied (beta $=-.26, p<.001$, in einer multiplen Regression von Depressivität auf verschiedene unabhängige Variablen). Für die seelische Gesundheit nach einer Scheidung scheinen somit Bildung und soziale Bindung gewichtige moderierende Faktoren zu sein: „The benefits of having a confidant and of regular social contact in terms of depressive feelings are also more pronounced in the divorced than in the continuously married population“ (S. 208). Auch wenn sich die dort betrachteten „Netzwerkmaße“ lediglich auf die subjektive Einschätzung der Einbettung in nachbarschaftliche Kontakte (,how close the respondent feels to people in the local area“, S. 203) sowie die Häufigkeit der Treffen mit Freunden, Verwandten oder Kollegen beziehen (,how often the respondent meets with friends, relatives, or colleagues", S. 203), so sind dies doch relevante Aspekte sozialer Netzwerke, die zum einen über die 
üblichen Supportfragen hinausweisen und zum anderen interessante ModeratorEffekte in Bezug auf sozioökonomischen Status und seelische Gesundheit zeigen.

\section{$6 \quad$ Fazit und Desiderata}

Die referierten Studien zeigen, dass es einerseits in der Forschung das Bemühen gibt, die black box „Netzwerke“ in ihrer Bedeutung für gesundheitliche Ungleichheiten genauer zu entschlüsseln, dass andererseits jedoch über die basale Unterscheidung von wahrgenommener Unterstützung oder der Größe von Familien- oder Freundschaftssektoren hinaus noch erheblicher Forschungsbedarf besteht. Es zeigte sich bislang kein einheitliches Bild zum Zusammenhang zwischen sozialen und gesundheitlichen Ungleichheiten sowie sozialen Netzwerke oder Transitionen im jungen und mittleren Erwachsenenalter.

Überwiegend bezieht sich die aktuelle empirische Forschung hierzu auf die oben in Abb. 1 skizzierten Moderator- oder Mediatormodelle. Dies erscheint uns auch vor dem Hintergrund der einleitenden Netzwerktheorien - als ein lohnenswerter und theoretisch gerechtfertigter Forschungsansatz, den es weiter zu verfolgen gilt. Denn in den meisten Theorien erfüllen Netzwerke genau dann ihre besten Aufgaben, wenn sie aus den passenden Netzwerkpartnern und -strukturen für eine spezifische Lebensphase oder Transition bestehen. Es lässt sich annehmen, dass womöglich diese Lebensphasen/Übergangs-Netzwerk-Passung den Ausschlag für das Eintreten (oder das Ausbleiben) gesundheitsförderlicher Effekte bildet. Einige Studien zeigten bereits, dass beispielsweise Freundes- oder Familiensektoren des Netzwerks ganz unterschiedliche Wirkungen auf Gesundheit haben können. Diese sektoralen Effekte gilt es weiter zu präzisieren und zu beforschen.

Dabei scheint die gesundheitliche Dynamik des Erwachsenenalters der Forschungsliteratur zufolge insgesamt weniger stark von eher langsamen biologischen Veränderungen herzurühren als aus einer Vielzahl zu bewältigender Übergänge, die sowohl mit ökonomischen (Ungleichheit) als auch sozialen Risiken (z. B. Netzwerkverkleinerung) verknüpft sind. In der Entwicklungspsychologie gibt es dazu eine Anzahl spezifischer Arbeiten, jedoch nur wenige übergreifende Theorien, die geeignet sind, diese Lebensphase als Ganzes zu beschreiben. Sowohl die Theorie der sozioemotionalen Selektivität als auch die Konvoi-Theorie (siehe Abschn. 2) gehen aber von einer übergreifenden Entwicklungsaufgabe aus - der Konstruktion von Lebensphasen-adäquaten sozialen Netzwerken. Das Gelingen dieser Konstruktion würde den Theorien zufolge eher mit einer günstigeren 
Prognose für (nicht nur seelische) Gesundheit einhergehen; das Misslingen eher mit einer ungünstigeren Prognose (z. B. Einsamkeit, schwache Unterstützung etc.). Ungleichheitsdimensionen werden hier allerdings oftmals nicht ausreichend berücksichtigt, ebenso wenig wie die Netzwerke hinreichend genau beschrieben werden.

Optimistisch für die weitere Forschung stimmt uns, dass die fraglichen Zusammenhäng in ihrer Komplexität zunehmend gesehen werden. So betonen Alwin et al. (2018a) in der Einleitung des aktuellen Bandes zu „Social Networks and the Life Course“, dass ,the understanding of social networks can improve the understanding of the life course, and vice versa." (S. 4). Dass diese wechselseitige Befruchtung auch Implikationen für gesundheitliche Ungleichheiten aufweist, wird jedoch nicht thematisiert. In der fast zeitgleich erschienenen Einleitung des Bandes „Life Course Health Development“ stellen Halfon et al. (2018a) das ,emerging field of life course health development“" (S. 2) vor, in dem wiederum gesundheitliche Ungleichheiten, nicht jedoch soziale Beziehungskontexte Erwähnung finden. Der Dreiklang aus Lebenslauf, Beziehungsnetzen und gesundheitlichen Ungleichheiten stellt somit eine logische Weiterentwicklung vorliegenden Wissens dar.

Studien, die diese komplexe Dynamik im Erwachsenenalter abbilden könnten, sollten daher verschiedenen Anforderungen genügen, von denen hier einige aufgelistet werden. Wir beginnen mit sieben methodischen Anforderungen, um dann auf die besprochenen Transitionen einzugehen.

1. Grundsätzlich ist zu bemängeln, dass sich kaum eine der vorgestellten Studien auf eine der hier ebenfalls vorgestellten Theorien bezieht. Jede der eingangs vorgestellten entwicklungspsychologischen Theorien hat unserer Meinung nach das Potenzial, Hypothesen bezüglich der diskutierten Zusammenhänge zu generieren. Alle Entwicklungstheorien sind ihrerseits in ein bio-psycho-soziales Modell eingebettet, in dem komplexe kausale Zusammenhänge gelten.

2. Daraus ergibt sich für uns, dass die Studien interdisziplinär in der Anlage sein sollten. Dabei gilt, dass alle Faktoren - auch biologische - veränderlich sein können und dementsprechend modelliert werden sollten. Beispielsweise sollten die Stichproben groß genug sein, um auch seltenere phasenspezifische gesundheitliche Übergänge (etwa Ansteckungen mit sexuell übertragbaren Krankheiten, etwa nach der Trennung einer Partnerschaft) so modellieren zu können, dass sich prämorbide oder distale Faktoren (z. B. Netzwerkveränderungen) hinreichend (im Vergleich zu getrennten Erwachsenen ohne Infektion) modellieren lassen. Hier ist die Zusammenarbeit von SoziologInnen und MedizinerInnen (ideal: wiederholte Reihenuntersuchungen) vonnöten. Bei der Planung der Messintervalle ist zu berücksichtigen, dass einige Faktoren in der Zeit nicht 
unmittelbar wirken, sondern ihre Wirkung erst nach einer gewissen Dauer entfalten. Beispielsweise wirkt sich allostatischer Stress durch den Verlust sozialer Beziehungen erst nach einiger Zeit und in Abhängigkeit von individuellen Bewältigungsmustern und Opportunitäten (etwa Nachbarschaftseffekte) aus.

3. Die Studien sollten vermehrt längsschnittlich angelegt sein, da nur auf diese Weise kausale Mechanismen im Zusammenhang von Ungleichheit, Netzwerk, Transition und Gesundheit analysiert werden können. Fragestellungen sollten grundsätzlich bidirektional und komplex gestellt werden, z. B. ob schlechte körperliche Gesundheit die Zugehörigkeit zu einer niedrigeren Einkommensklasse prädiziert oder vice versa. Netzwerke können in komplexen längsschnittlichen Modellen sowohl als Ursachen, Moderatoren oder Mediatoren, aber auch als abhängige Variable betrachtet werden.

4. Die Stichproben sollten groß genug sein, um ausreichend Varianz sowohl auf den Ungleichheitsdimensionen als auch in der Netzwerkentwicklung abbilden zu können. Proximale Faktoren, wie konstitutionelle physische Ressourcen ${ }^{5}$, Gesundheitsverhalten, Persönlichkeit oder elterliche Erziehungspraktiken erklären große Teile gesundheitlicher Ungleichheit, womit für den Nachweis der Wirkung eher distaler Faktoren Stichproben erforderlich sind, die MehrEbenen-Analysen erlauben. Um beispielsweise Einflüsse des Netzwerkes von denen sozialer Ungleichheit zu trennen, sind große Stichproben innerhalb einer Einkommensgruppe nötig, in denen die Netzwerkparameter hinreichend variieren, wobei andere Einflüsse kontrolliert werden sollten.

5. Da sich sozialer Wandel erheblich auf die Art und den Zeitpunkt der Übergänge auswirkt, sollte er Teil des Modells und der Stichprobenplanung sein. Letzteres wird durch cross-sequenzielle Designs erreicht, in denen unterschiedliche Alterskohorten für verschiedene Wandeleffekte stehen.

6. Studien zum Erwachsenenalter sollten möglichst genau den Zeitpunkt (damit auch die Sequenz) und den Kontext von Transitionen (als zeitabhängige Kovariaten) erfassen, ebenso wie den objektiven (beispielsweise den Einkommensverlust oder einen Wohnortwechsel) und subjektiven (beispielsweise Stress und Kausalattribution) Einfluss der Übergänge.

\footnotetext{
${ }^{5}$ Gemeint sind vor allem genetische Ressourcen. Deren Einbezug in interdisziplinäre Studien verlangt nach genetisch informativen Designs bei gleichzeitiger Erhebung klassischer soziologischer Indikatoren in großen Stichproben. Ein Beispiel für derartige Forschung in Deutschland ist die TwinLife Studie (http://www.twin-life.de/de), die bisher leider nicht das mittlere Erwachsenenalter untersucht.
} 
7. Soziale Netzwerke sollten hinreichend gründlich erfasst werden. Den hier besprochenen Studien ist gemein, dass entweder nur grobe Schätzer (beispielsweise zur Anzahl der Freunde, Kontaktintensitäten) oder nur bereichsspezifische Schätzer (beispielsweise nur egozentrierte Netzwerke) erhoben wurden. Die Frage, inwieweit sich mit den Übergängen des Erwachsenenalters auch Veränderungen im weiteren Netzwerk, etwa in den Beziehungen der Alteri untereinander, ergeben, konnte in keiner der referierten Studien beantwortet werden. Die Beziehungen im weiteren Netzwerk sollten jedoch als Teil des sozialen Kapitals einer Person betrachtet werden; auch unterbrochene Dyaden in Alteri-Beziehungen können transitionsbedingt gesundheitliche oder ökonomische Konsequenzen haben ${ }^{6}$. Hier gilt es, vorhandene theoretische Ansätze auf das gesamte Netzwerk auszuweiten.

Den Autoren ist bisher keine Studie bekannt, die all diese Anforderungen erfüllen könnte. Neben diesen allgemeinen Kritikpunkten gibt es zu den einzelnen Transitionen weitere offene Forschungsfragen, von denen einige angesprochen werden sollen.

Die Empty-Nest-Transition ist in ihrer Wirkung auf das Netzwerk bisher für Industriegesellschaften wenig differenziert dargestellt. Da beispielsweise in Deutschland gebildetere Mütter ihre Kinder in höherem Alter als bildungsfernere bekommen und dann aufgrund längerer Schulzeiten länger in der Familie halten, müssten sie älter sein, womit für sie seltener eine Phase der ,zweiten Jugend“ und der zugehörigen Netzwerkvergrößerung in Betracht kämen. Hier wird eine besondere Interaktion zwischen biologischem Alter, Bildungsstand und Transition deutlich, denn Individuen, die beispielsweise erst mit 40 Jahren Eltern werden, dürften die Empty-Nest-Situation erst um das sechzigste oder fünfundsechzigste Lebensjahr erleben (inkl. dem entsprechenden Risiko für späte Scheidungen). In diesem Lebensalter sind dann jedoch auch wiederum Lebensalter-spezifische Verkleinerungen des Netzwerkes eher erwartbar (siehe Kap., ,Soziale Netzwerke und gesundheitliche Ungleichheiten im Alter"). Auf der anderen Seite dürften finanzielle Ressourcen im Zuge des sozialen Wandels (Zunahme der Einkommensschere) für ärmere Nestflüchter geringer werden, womit hier

${ }^{6}$ Fällt beispielsweise der Auszug der Kinder mit der Trennung der Eltern zusammen, wird die Nutzung elterlicher „Beziehungen“ zu entfernten Netzwerkpersonen durch die ausziehenden Kinder, etwa für Ausbildung oder Wohnung, wohlmöglich weniger wahrscheinlich. Es ließe sich auch sagen, dass die Trennung der Eltern die Verfügbarkeit ihres „,second-hand social capital“ (Shah et al. 2018) für die Kinder verringert. 
crowding-in-Phänomene und unfreiwillig verlängerte Eltern-Kind-Phasen wahrscheinlicher werden. Inwieweit hier z. B. enttäuschte elterliche Erwartungen auf eine „Zweite Jugend“ (keine erneute Partnerschaft oder Elternschaft, da sich das Netzwerk ohne Auszug der Kinder nicht erweitert) gerade bei Ressourcenknappheit zu einer Verschärfung gesundheitlicher Probleme führt, bleibt eine der offenen Fragestellungen - auch in den ungleicher werdenden Industriegesellschaften. Diese hier nur angedeuteten und komplexen Wechselwirkungen zwischen den in Abschn. 1.2 skizzierten Themen soll hier nur eine von zahlreichen Forschungsdesiderata illustrieren.

Ein ähnlicher Präzisierungsbedarf in Bezug auf mögliche Wechselwirkungen lässt sich in Bezug auf Heirat und/oder Scheidung konstatieren. Während die Arbeiten der Forschungsgruppen um Felmlee (2001) und Sprecher et al. (2006) zeigen können, dass und in welcher Form Netzwerke das Paarleben fortwährend begleiten, so bleiben doch die Fragen unbeantwortet, ob hiermit stets auch soziale und gesundheitliche Ungleichheiten einhergehen. Spielen die sozialen Netzwerke von Ungebundenen, Paaren oder Geschiedenen für alle Formen der psychischen und physischen Gesundheit sowie für die Reproduktion von gesundheitlicher Ungleichheiten dieselbe Rolle oder lohnt sich hier der genauere Blick? Auch hierauf weiß die Forschung derzeit keine abschließende Antwort.

Aus der Ungleichheitsperspektive liegt für uns jedoch der Gedanke des Matthäus-Prinzips nahe: Individuen, die bereits zu Beginn ihrer Lebensspanne in gesünderen, beziehungszufriedeneren und ressourcenstärkeren Netzwerken leben, werden womöglich im Lebenslauf auch dadurch noch etwas gesünder, beziehungszufriedener und ressourcenstärker werden als andere. Für die weitere Untersuchung des Wechselspiels dieser Faktoren und für die Gesundheitsprävention sind dies allerdings relevante Ansatzpunkte.

\section{Leseempfehlungen}

Alwin, D. F., Felmlee, D. H., \& Kreager, D. A. (Hrsg.) (2018). Social Networks and the Life Course: Integrating the Development of Human Lives and Social Relational Networks. Cham, CH: Springer. Dieser aktuelle Sammelband stellt den Stand und die Perspektiven der innovativen Synthese aus Lebenslauf- und Netzwerkforschung in 22 Kapiteln vor.

Halfon, N., Forrest, C.B., Lerner, R.M. \& Faustman, E.M. (Hrsg.) (2018). Handbook of life course health development. Cham, CH: Springer Nature. Dieser aktuelle Sammelband stellt den Stand und die Perspektiven des neuen Forschungsbereichs LCHD (Life Course Health Development) in 26 Kapiteln vor. 
Kalmijn, M. (2003). Shared friendship networks and the life course: An analysis of survey data on married and cohabiting couples. Social Networks, 25, S. 231-249. Diese Studie erfüllt bereits viele der hier erhobenen Forderungen, denn sie ist orientiert am gesamten Lebenslauf des Erwachsenenalters und beschreibt acht verschiedene Transitionen in ihren Assoziationen mit dem Freundesnetzwerk an einer großen Stichprobe $(N=2977)$. Sie ist theoretisch orientiert (unterbrochene Dyaden, Balance-Prinzip) und berücksichtigt querschnittlich diverse sozioökonomische Variablen, ohne allerdings auf Gesundheit im engeren Sinne zu fokussieren. Damit kann sie keine Aussagen zu Veränderung gesundheitlicher Ungleichheit machen, gibt aber eine gute Orientierung für weitere Forschung.

Wrzus, C., Hänel, M., Wagner, J., \& Neyer, F. J. (2013). Social network changes and life events across the life span: A meta-analysis. Psychological Bulletin, 139 (1), S. 53-80. Eine maßgebliche Meta-Analyse der Veränderung egozentrierter Beziehungsnetze von der Kindheit bis zur Seneszenz mit einer großen Anzahl an Primärstudien $(k=243)$.

\section{Datensätze}

- „Allgemeine Bevölkerungsumfrage der Sozialwissenschaften“ (ALLBUS).

Seit 1980 werden in der Regel alle zwei Jahre repräsentative Daten über Einstellungen, Verhaltensweisen und Sozialstruktur der Bevölkerung in der Bundesrepublik Deutschland querschnittlich erhoben. Die Datenbank enthält einige Indikatoren zu sozialer Ungleichheit, Gesundheit und Gesundheitsverhalten, sozialen Netzwerken und sozialem Kapital. Weitere Informationen: http://www.gesis.org/allbus/allbus

- Studien aus dem Gesundheitsmonitoring des Robert-Koch-Instituts: „Studie zur Gesundheit Erwachsener in Deutschland“ (DEGS) und ,Gesundheit in Deutschland aktuell“" (GEDA)

In einer Kombination aus quer- und längsschnittlichen (DEGS) sowie querschnittlichen Daten (GEDA) werden seit 2008 in zweijährigem Rhythmus umfangreiche repräsentative Gesundheitsdaten der deutschen Wohnbevölkerung erhoben, die teilweise ärztliche Untersuchungsdaten umfassen. Es sind in unterschiedlichem Umfang verschiedene 
Indikatoren zur sozialen Ungleichheit sowie zur sozialen Unterstützung enthalten. Weitere Informationen: http://www.degs-studie.de und http:// www.geda-studie.de

- „Sozio-oekonomisches Panel“ (SOEP)

Jährliche, längsschnittliche Repräsentativbefragung der erwachsenen Bevölkerung Deutschlands (fortlaufend seit 1984 bzw. 1990 in Westbzw. Ostdeutschland). Fokussiert auf die soziale und ökonomische Situation der Bevölkerung, inkludiert aber regelmäßig auch psychologische und Netzwerk-Indikatoren in die Erhebung. Weitere Informationen: http//www.diw.de/de/soep

\section{Literatur}

Alwin, D. F., Felmlee, D. H., \& Kreager, D. A. (2018a). Together through time. Social networks and the life course. In D. F. Alwin, D. H. Felmlee, \& D. A. Kreager (Hrsg.), Social networks and the life course. Integrating the development of human lives and social relational networks (S. 3-26). Cham: Springer.

Alwin, D. F., Felmlee, D. H., \& Kreager, D. A. (Hrsg.). (2018b). Social networks and the life course. Integrating the development of human lives and social relational networks. Cham: Springer.

Antonucci, T. C., Fiori, K. L., Birditt, K., \& Jackey, L. M. H. (2010). Convoys of social relations: Integrating life-span and life-course perspectives. In M. E. Lamb, A. M. Freund, \& R. M. Lerner (Hrsg.), The handbook of life-span development (Bd. 2, S. 434473)., Social and emotional development Hoboken: Wiley.

Antonucci, T. C., Birditt, K. S., \& Ajrouch, K. (2011). Convoys of social relations. Past, present, and future. In K. L. Fingerman, C. A. Berg, J. Smith, \& T. C. Antonucci (Hrsg.), Handbook of life-span development (S. 161-182). New York: Springer.

Baltes, P. B. (1987). Theoretical propositions of life-span developmental psychology. On the dynamics between growth and decline. Developmental Psychology, 23(5), 611-626.

Baltes, P. B., \& Smith, J. (2004). Lifespan psychology. From developmental contextualism to developmental biocultural co-constructivism. Research in Human Development, 1(3), 123-144.

Beaupré, P., Turcotte, P., \& Milan, A. (2006). When is junior moving out? Transitions from the parental home to independence. Canadian Social Trends, 82, 9-15.

Becker, P. (2006). Gesundheit durch Bedürfnisbefriedigung. Göttingen: Hogrefe.

Blanchflower, D. G., \& Oswald, A. (2017). Do humans suffer a psychological low in midlife? Two approaches (with and without controls) in seven data sets. Cambridge: National Bureau of Economic Research. 
Bouchard, G. (2014). How do parents react when their children leave home? An integrative review. Journal of Adult Development, 21, 69-79.

Brandtstädter, J., \& Lindenberger, U. (Hrsg.). (2007). Entwicklungspsychologie der Lebensspanne. Stuttgart: Kohlhammer.

Burger, E., \& Milardo, R. M. (1995). Marital interdependence and social networks. Journal of Social and Personal Relationships, 12(3), 403-415.

Cable, N., Bartley, M., Chandola, T., \& Sacker, A. (2013). Friends are equally important to men and women, but family matters more for men's well-being. Journal of Epidemiology and Community Health, 67(2), 166-171.

Carr, D., \& Springer, K. W. (2010). Advances in families and health research in the $21 \mathrm{st}$ century. Journal of Marriage and Family, 72(3), 743-761.

Carstensen, L. L., Isaacowitz, D. M., \& Charles, S. T. (1999). Taking time seriously. A theory of socioemotional selectivity. American Psychologist, 54(3), 165-181.

Chappell, N. L., \& Funk, L. M. (2010). Social capital. Does it add to the health inequalities debate? Social Indicators Research, 99(3), 357-373.

Costa, P. J., \& McCrae, R. R. (1994). Set like plaster. Evidence for the stability of adult personality. In T. E. Heatherton \& J. L. Weinberger (Hrsg.), Can personality change?. Washington: American Psychological Association.

Cutler, D. M., \& Lleras-Muney, A. (2010). Understanding differences in health behaviors by education. Journal of Health Economics, 29(1), 1-28.

Dalgard, O. S., \& Håheim, L. (1998). Psychosocial risk factors and mortality. a prospective study with special focus on social support, social participation, and locus of control in Norway. Journal of Epidemiology and Community Health, 52(8), 476-481.

Dragano, N. (2007). Arbeit, Stress und krankheitsbedingte Frührenten. Zusammenhänge aus theoretischer und empirischer Sicht. Wiesbaden: Springer VS.

Ertel, K. A., Glymour, M. M., \& Berkman, L. F. (2009). Social networks and health: A life course perspective integrating observational and experimental evidence. Journal of Social and Personal Relationships, 26(1), 73-92.

Esmaeili, N. S., Yaacob, S. N., Juhari, R., \& Schoebi, D. (2015). Predictors of psychological distress among divorced women in Iran. Journal of Divorce \& Remarriage, 56(3), 248-259.

Felmlee, D. H. (2001). No couple is an island. A social network perspective on dyadic stability. Social Forces, 79(4), 1259-1287.

Filipp, S.-H., \& Aymanns, P. (1987). Die Bedeutung sozialer und personaler Ressourcen in der Auseinandersetzung mit kritischen Lebensereignissen. Zeitschrift für Klinische Psychologie, 16(4), 383-396.

Fooken, I., \& Kavšek, N. (2012). Entwicklungspsychologische Grundlagen. In E. Brähler \& B. Strau (Hrsg.), Grundlagen der Medizinischen Psychologie (S. 251-283). Göttingen: Hogrefe.

Halfon, N., Forrest, C. B., Lerner, R. M., Faustman, E. M., Tullis, E., \& Son, J. (2018a). Introduction to the handbook of life course health development. In N. Halfon, C. B. Forrest, R. M. Lerner, \& E. M. Faustman (Hrsg.), Handbook of life course health development (korr. Druck, S. 1-16). Cham: Springer Nature. 
Halfon, N., Forrest, C. B., Lerner, R. M., \& Faustman, E. M. (Hrsg.). (2018b). Handbook of life course health development. Cham: Springer Nature.

Hansen, F. J., Fallon, A. E., \& Novotny, S. L. (1991). The relationship between social network structure and marital satisfaction in distressed and nondistressed couples. A pilot study. Family Therapy, 18(2), 101-114.

Harkins, E. B. (1978). Effects of empty nest transition on self-report of psychological and physical well-being. Journal of Marriage and the Family, 40(3), 549-556.

Havighurst, R. J. (1976). Developmental tasks and education. New York: McKay.

Hoffmann, R., Kröger, H., \& Geyer, S. (2018). Social causation versus health selection in the life course. Does their relative importance differ by dimension of SES? Social Indicators Research, 141, 1341. https://doi.org/10.1007/a:112050181871.

Hughes, R., Good, E. S., \& Candell, K. (1993). A longitudinal study of the effects of social support on the psychological adjustment of divorced mothers. Journal of Divorce \& Remarriage, 19(1-2), 37-56.

Hurrelmann, K., \& Richter, M. (2013). Gesundheits- und Medizinsoziologie. Eine Einführung in sozialwissenschaftliche Gesundheitsforschung. Weinheim: Beltz Juventa.

Johnson, M. P., \& Leslie, L. (1982). Couple involvement and network structure. A test of the dyadic withdrawal hypothesis. Social Psychology Quarterly, 45(1), 34-43.

Kahn, R. L., \& Antonucci, T. C. (1980). Convoys over the life course. Attachment, roles and social support. In P. B. Baltes \& O. G. J. Brim (Hrsg.), Life-span development and behavior (Bd. 3, S. 253-286). New York: Academic.

Kalmijn, M. (2003). Shared friendship networks and the life course. An analysis of survey data on married and cohabiting couples. Social Networks, 25, 231-249.

Kalmijn, M., \& Bernasco, W. (2001). Joint and separated lifestyles in couple relationships. Journal of Marriage and Family, 63(3), 639-654.

Kalmijn, M., \& van Groenou, M. B. (2005). Differential effects of divorce on social integration. Journal of Social and Personal Relationships, 22(4), 455-476.

Kincaid, S. B., \& Caldwell, R. A. (1991). Initiator status, family support, and adjustment to marital separation. A test of an interaction hypothesis. Journal of Community Psychology, 19(1), 79-88.

Klauer, T., \& Greve, W. (2005). Kritische Lebensereignisse und Gesundheit. In R. Schwarzer (Hrsg.), Gesundheitspsychologie (S. 237-259). Göttingen: Hogrefe.

Kleinepier, T., Berrington, A., \& Stoeldraijer, L. (2017). Ethnic differences in returning home. Explanations from a life course perspective. Journal of Marriage and Family, 79, 1023-1040.

Knoll, N., \& Schwarzer, R. (2005). Soziale Unterstützung. In R. Schwarzer (Hrsg.), Gesundheitspsychologie (S. 333-349). Göttingen: Hogrefe.

Knoll, N., Scholz, U., \& Rieckmann, N. (2011). Einführung Gesundheitspsychologie. München: Reinhardt UTB.

Kupek, E. (2001). Sexual attitudes and number of partners in young British men. Archives of Sexual Behavior, 30(1), 13-27.

Lachman, M. E., Teshale, S., \& Agrigoroaei, S. (2015). Midlife as a pivotal period in the life course. Balancing growth and decline at the crossroads of youth and old age. International Journal of Behavioral Development, 39(1), 20-31.

Lang, F. R., Reschke, F. S., \& Neyer, F. J. (2006). Social relationships, transitions, and personality development across the life-span. In D. K. Mroczek \& T. D. Little (Hrsg.), Handbook of personality development (S. 445-467). Mahwah: Erlbaum. 
Leopold, T. (2018). Gender differences in the consequences of divorce. A study of multiple outcomes. Demography, 55(3), 769-797.

Levitt, M. J., Weber, R. A., \& Clark, M. C. (1986). Social network relationships as sources of maternal support and well-being. Developmental Psychology, 22(3), 310-316.

Lillard, L. A., \& Waite, L. J. (1995). Til death do us part. Marital disruption and mortality. American Journal of Sociology, 100(5), 1131-1156.

Liu, L.-J., \& Guo, Q. (2008). Life satisfaction in a sample of empty-nest elderly. A survey in the rural area of a mountainous county in China. Quality of Life Research, 17, $823-830$.

Lohaus, A., \& Klein-Heßling, J. (2009). Besondere Lebensabschnitte. In J. Bengel \& M. Jerusalem (Hrsg.), Handbuch der Gesundheitspsychologie und Medizinischen Psychologie (S. 164-171). Göttingen: Hogrefe.

Marmot, M. G. (2000). Multilevel approaches to understanding social determinants. In L. F. Berkman \& I. Kawachi (Hrsg.), Social epidemiology (S. 349-369). Oxford: University Press.

Mayer, K. U. (2000). Promises fulfilled? A review of 20 years of life course research. European Journal of Sociology, 41(2), 259-282.

Mielck, A. (2005). Soziale Ungleichheit und Gesundheit. Einführung in die aktuelle Diskussion. Bern: Huber.

Milardo, R. M. (1989). Theoretical and methodological issues in the identification of the social Networks of spouses. Journal of Marriage and Family, 51(1), 165-174.

Milardo, R. M., \& Allan, G. (2000). Social networks and marital relationships. In R. M. Milardo \& S. Duck (Hrsg.), Families as relationships (S. 117-134). New York: Wiley.

Millstein, S. G., Moscicki, A.-B., \& Broering, J. M. (1994). Female adolescents at high, moderate, and low risk of exposure to HIV. Differences in knowledge, beliefs, and behavior. Journal of Adolescent Health, 15(2), 133-141.

Mínguez, A. M. (2016). Late leaving of the parental home in Southern Europe. Lessons for Youth Policy. Comparative Sociology, 15(4), 485-507.

Morris, M., \& Kretzschmar, M. (1995). Concurrent partnerships and transmission dynamics in networks. Social Networks, 17(3-4), 299-318.

Murphy, S. A., Lohan, J., Dimond, M., \& Fan, J. (1998). Network and mutual support for parents bereaved following the violent deaths of their 12- to 28-year old children. A longitudinal prospective analysis. Journal of Personal \& Interpersonal Loss, 3(4), 303-333.

Nagy, M. E., \& Theiss, J. A. (2013). Applying the relational turbulence model to the empty-nest transition. Sources of relationship change, relational uncertainty and interference from partners. Journal of Family Communication, 13, 280-330.

Neyer, F. J., \& Asendorpf, J. B. (2001). Personality-relationship transaction in young adulthood. Journal of Personality and Social Psychology, 81(6), 1190-1204.

Neyer, F. J., Mund, M., Zimmermann, J., \& Wrzus, C. (2014). Personality-relationship transactions revisited. Journal of Personality, 82(6), 539-550.

Papastefanu, C. (2000). Der Auszug aus dem Elternhaus. Ein vernachlässigter Gegenstand der Entwicklungspsychologie. Zeitschrift für Soziologie der Erziehung und Sozialisation, 20(1), 55-69.

Pollmann-Schult, M. (2011). Soziale Integration und Lebenszufriedenheit kinderloser Frauen und Männer im mittleren und höheren Erwachsenenalter. Zeitschrift für Gerontologie und Geriatrie, 44, 411-416. 
Riggs, B. L., Melton, L. J., Robb, R. A., Camp, J. J., Atkinson, E. J., McDaniel, L., Amin, S., Rouleau, P. A., \& Khosla, S. (2008). A Population-based assessment of rates of bone loss at multiple skeletal sites. Evidence for substantial trabecular bone loss in young adult women and men. Journal of Bone and Mineral Research, 23(2), 205-214.

Rissel, C., Badcock, P. B., Smith, A. M. A., Richters, J., de Visser, R. O., Grulich, A. E., \& Simpson, J. M. (2014). Heterosexual experience and recent heterosexual encounters among Australian adults. The second Australian study of health and relationships. Sexual Health, 11(5), 416-426.

Roberts, B. W. (1997). Plaster or plasticity: Are adult work experiences associated with personality change in women? Journal of Personality, 65(2), 205-232.

Roberts, B. W., \& DelVecchio, W. F. (2000). The rank order consistency of personality from childhood to old age. A quantitative review of longitudinal studies. Psychological Bulletin, 126, 3-25.

Rubin, M., Evans, O., \& Wilkinson, R. B. (2016). A longitudinal study of the relations among university students' subjective social status, social contact with university friends, and mental health and well-being. Journal of Social and Clinical Psychology, 35(9), 722-737.

Santrock, J. W. (2015). Life-span development. New York: McGraw-Hill.

Schneider, N. F., \& Rüger, H. (2008). Beziehungserfahrungen und Partnerschaftsverläufe vor der Heirat. Eine empirische Analyse von Angehörigen der Eheschließungskohorte 1999-2005. Zeitschrift für Familienforschung, 20(2), 131-156.

Schütz, A., \& Wiesner, C. (2000). Partnerschaft und Gesundheitszustand. In P. Kaiser (Hrsg.), Partnerschaft und Paartherapie (S. 193-218). Göttingen: Hogrefe.

Schwartz, C. R., \& Han, H. (2014). The reversal of the gender gap in education and trends in marital dissolution. American Sociological Review, 79(4), 605-629.

Schwarzer, R., \& Leppin, A. (1989). Social support and health. A meta-analysis. Psychology \& Health, 3(1), 1-15.

Shah, N. P., Levin, D. Z., \& Cross, R. (2018). Secondhand social capital. Boundary spanning, secondhand closure, and individual performance. Social Networks, 52, 18-27.

Smith, C. E., Fernengel, K., Holcroft, C., \& Gerald, K. (1994). Meta-analysis of the associations between social support and health outcomes. Annals of Behavioral Medicine, $16(4), 352-362$.

Solomon, D. H., \& Knobloch, L. K. (2004). A model of relational turbulence. The role of intimacy, relational uncertainty, and interference from partners in appraisals of irritations. Journal of Social and Personal Relationships, 21(2), 795-816.

Soons, J. P. M., Liefbroer, A. C., \& Kalmijn, M. (2009). The long-term consequences of relationship formation for subjective well-being. Journal of Marriage and Family, 71(5), 1254-1270.

South, S. J., \& Lei, L. (2015). Failures-to-launch and boomerang kids. Contemporary determinants of leaving and returning to the parental home. Social Forces, 94(2), 863890.

Specht, J., Bleidorn, W., Denissen, J. J. A., Hennecke, M., Hutteman, R., Kandler, C., Luhmann, M., Orth, U., Reitz, A. K., \& Zimmermann, J. (2014). What drives adult personality development? A comparison of theoretical perspectives and empirical evidence. European Journal of Personality, 28(3), 216-230. 
Sprecher, S., Felmlee, D., Schmeeckle, M., \& Shu, X. (2006). No breakup occurs on an island. Social networks and relationship dissolution. In M. A. Fine \& J. H. M. E. Harvey (Hrsg.), Handbook of divorce and relationship dissolution (S. 457-478). Mahwah: Erlbaum.

Steptoe, A., \& Marmot, M. (2003). Burden of psychosocial adversity and vulnerability in middle age. Associations with biobehavioral risk factors and quality of life. Psychosomatic Medicine, 65(6), 1029-1037.

Stone, G. (2002). Nonresidential father postdivorce well-being. The role of social supports. Journal of Divorce \& Remarriage, 36(3-4), 139-150.

Stone, A. A., Schwartz, J. E., Broderick, J. E., \& Deaton, A. (2010). A snapshot of the age distribution of psychological well-being in the United States. PNAS Proceedings of the National Academy of Sciences of the United States of America, 107(22), 9985-9990.

Sun, K., Zuo, M., \& Kong, D. (2016). Communication with children for older adults' life satisfaction. Non-face-to-face and/or face-to-face? Gerontechnology, 14(4), 191-203.

Sussman, S., Lisha, N., \& Griffiths, M. (2011). Prevalence of the addictions. A problem of the majority or the minority? Evaluation \& the Health Professions, 34(1), 3-56.

Symoens, S., Van de Velde, S., Colman, E., \& Bracke, P. (2014). Divorce and the multidimensionality of men and women's mental health. The role of social-relational and socio-economic conditions. Applied Research in Quality of Life, 9(2), 197-214.

Tanfer, K., Cubbins, L. A., \& Billy, J. O. (1995). Gender, race, class and self-reported sexually transmitted disease incidence. Family Planning Perspectives, 27(5), 196-202.

Tanis, M., van der Louw, M., \& Buijzen, M. (2017). From empty nest to Social Networking Site. What happens in cyberspace when children are launched from the parental home? Computers in Human Behavior, 68, 56-63.

Terhell, E. L., Broese van Groenou, M. I., \& van Tilburg, T. (2004). Network dynamics in the long-term period after divorce. Journal of Social and Personal Relationships, 21(6), 719-738.

Valente, T. W. (2010). Social networks and health. Models, methods and applications. Oxford: University Press.

Van Dulmen, M. H. M. (2013). Emerging Adulthood. The Journal. Emerging Adulthood, $1(1), 3-4$.

Veiel, H. O., Crisand, M., Stroszeck-Somschor, H., \& Herrle, J. (1991). Social support networks of chronically strained couples. Similarity and overlap. Journal of Social and Personal Relationships, 8(2), 279-292.

Vonneilich, N., Jöckel, K.-H., Erbel, R., Klein, J., Dragano, N., Siegrist, J., \& von dem Knesebeck, O. (2012). The mediating effect of social relationships on the association between socioeconomic status and subjective health. Results from the Heinz Nixdorf Recall cohort study. BMC Public Health, 12, 285.

Wan, H., Yu, F., \& Kolanowski, A. (2008). Caring for aging chinese. Lessons learned from the United States. Journal of Transcultural Nursing, 19(2), 114-120.

Wayment, H. A., Wyatt, G. E., Tucker, M. B., Romero, G. J., Carmona, J. V., Newcomb, M., Solis, B. M., Riederle, M., \& Mitchell-Kernan, C. (2003). Predictors of risky and precautionary sexual behaviors among single and married white women. Journal of Applied Social Psychology, 33(4), 791-816.

Weber, H. (2005). Persönlichkeit und Gesundheit. In R. Schwarzer (Hrsg.), Gesundheitspsychologie (S. 129-147). Göttingen: Hogrefe. 
Widmer, E. D., Kellerhals, J., \& Levy, R. (2004). Types of conjugal networks, conjugal conflict and conjugal quality. European Sociological Review, 20(1), 63-77.

Wurm, S., \& Tesch-Römer, C. (2005). Alter und Gesundheit. In R. Schwarzer (Hrsg.), Gesundheitspsychologie (S. 71-90). Göttingen: Hogrefe.

Wrzus, C., Hänel, M., Wagner, J., \& Neyer, F. J. (2013). Social network changes and life events across the life span. A Meta-Analysis. Psychological Bulletin, 139(1), 53-80.

Yeung, D. Y., Fung, H. H., \& Lang, F. R. (2008). Self-construal moderates age differences in social network characteristics. Psychology and Aging, 23(1), 222-226.

Open Access Dieses Kapitel wird unter der Creative Commons Namensnennung 4.0 International Lizenz (http://creativecommons.org/licenses/by/4.0/deed.de) veröffentlicht, welche die Nutzung, Vervielfältigung, Bearbeitung, Verbreitung und Wiedergabe in jeglichem Medium und Format erlaubt, sofern Sie den/die ursprünglichen Autor(en) und die Quelle ordnungsgemäß nennen, einen Link zur Creative Commons Lizenz beifügen und angeben, ob Änderungen vorgenommen wurden.

Die in diesem Kapitel enthaltenen Bilder und sonstiges Drittmaterial unterliegen ebenfalls der genannten Creative Commons Lizenz, sofern sich aus der Abbildungslegende nichts anderes ergibt. Sofern das betreffende Material nicht unter der genannten Creative Commons Lizenz steht und die betreffende Handlung nicht nach gesetzlichen Vorschriften erlaubt ist, ist für die oben aufgeführten Weiterverwendungen des Materials die Einwilligung des jeweiligen Rechteinhabers einzuholen.

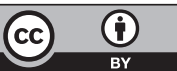

